\title{
Hydrogenated amorphous silicon characterization from steady state photoconductive measurements
}

\author{
Leonardo Kopprio ${ }^{1}$, Christophe Longeaud ${ }^{2}$ and Javier Schmidt ${ }^{1,3}$ \\ ${ }^{1}$ Instituto de Física del Litoral (IFIS-Litoral), CONICET-UNL, Güemes 3450, S3000GLN Santa Fe, Argentina \\ ${ }^{2}$ GEEPS, CNRS (UMR 8507), CentraleSupelec, UPSUd, UPMC, 11 Rue Joliot Curie, 91190 Gif sur Yvette, \\ France \\ ${ }^{3}$ Facultad de Ingeniería Química, Universidad Nacional del Litoral, Santiago del Estero 2829, S3000AOM \\ Santa Fe, Argentina
}

\begin{abstract}
We propose to use steady state measurements and a teaching-learning-based optimization (TLBO) algorithm to get the complete set of material transport parameters of disordered semiconductors, taking undoped hydrogenated amorphous silicon as an example. First, the steady-state conductivity under illumination and the ambipolar diffusion length $\left(L_{a m b}\right)$ are measured for several temperatures and generation rates. The steady-state photocarrier grating (SSPG) technique is used for the evaluation of $L_{a m b}$. Then, the TLBO algorithm is used for the obtainment of the material parameters that best satisfy the charge neutrality and the continuity equations. The use of this algorithm allowed us to get an excellent estimation of the valence band tail slope, as compared to the one obtained from measurements of the absorption coefficient by Fourier transform photocurrent spectroscopy and transmittance/reflectance. The dangling bonds and the conduction band tail parameters were also found to be in very good agreement with those measured from high frequency modulated photocurrent experiments (MPC). Numerical simulations show that the capture coefficients of the band tails and defects states can also be estimated, although with less precision than the DOS parameters.
\end{abstract}

\section{Introduction}

In the search of energy sources alternative to fossil fuels, photovoltaic devices based on disordered semiconductors are especially attractive due to their low cost and their direct band gap leading to a high absorption coefficient [1]. The most thoroughly studied disordered semiconductor is hydrogenated amorphous silicon (a-Si:H), which attracted considerable attention in the past and has currently many applications [2,3]. In a-Si:H, localized states are extremely important because they act as traps and recombination centers for the free carriers (i.e., those located in delocalized states). The localized states are energetically situated between the valence band edge $\left(E_{V}\right)$ and the conduction band edge $\left(E_{C}\right)$. It is usually assumed that the carrier mobility in localized states is null, and for that reason the energy region between the band edges is called the mobility gap [4]. The concept of a mobility gap was first introduced in Ref. [5], where the authors also propose band tails extending from the band edges towards the mobility gap, originated by the disorder of the structure. Nowadays, band tails are 
recognized as a common characteristic of disordered semiconductors $[3,6]$. In addition to the tail states, coordination defects also introduce dangling bond (DB) states, located around midgap. Hydrogen is extremely important in a-Si:H, since these atoms saturate silicon DBs and remove defects from the gap, although a density of around $10^{16} \mathrm{~cm}^{-3}$ defects remain even in device-quality material. Powell and Deane [7] and Schumm [8] developed a chemical equilibrium model that describes the hydrogen-mediated creation of defects in a-Si:H, generically known as Defect Pool Model. According to this model, the density of deep states consists in the sum of three Gaussian functions, corresponding to negatively charged, neutral and positively charged defects. The density of states (DOS) distribution within the mobility gap of a-Si:H, and the values of the parameters that describe the DOS, are still a matter of debate. These values are needed as input parameters for all those simulation programs dealing with the modeling and optimization of devices incorporating a-Si:H layers $[9,10]$.

Several experimental methods have been developed to obtain the DOS within the mobility gap of amorphous semiconductors exhibiting photoconductivity. These methods can be classified into two broad categories: (i) methods where the experimental data are used directly to determine the DOS, based on an approximate reconstruction formula derived from a theoretical analysis of the experiment $[11,12]$; and (ii) methods where an initial DOS described by several parameters is proposed, obtaining the parameter values from a fit of the experimental data with a formula provided by the theoretical description of the experiment $[6,13]$. The main drawback of the methods belonging to the first category is that several approximations are needed to obtain an analytical formula for the DOS, which is usually valid over only a limited energy range. Moreover, accepted values for the carrier mobilities and for the capture coefficients are usually assumed. The main drawback of the second group of methods is that no less than twenty parameters are involved in the description of the DOS, generating concerns about the uniqueness of the fit. However, the continuous growth of the calculation power of computers and the constant evolution of optimization algorithms increase the capability of the second group of methods, especially when a single set of parameter values is used to fit the results of several measurements.

In this work, we test the possibility of using the continuity and charge neutrality equations for the determination of the material parameters of undoped a-Si:H. The proposed methodology consists on first performing measurements of steady state conductivity under illumination and ambipolar diffusion length, for a wide range of temperatures $(T)$ and generation rates $(G)$, on thin film samples provided with coplanar ohmic contacts. From these measurements, the steady-state concentrations of free electrons and holes, $n(T, G)$ and $p(T, G)$, are determined for each condition. Then, a simplified Teaching-Learning-Based Optimization (TLBO) algorithm [14,15] is used for finding the set of material parameters that best satisfy the continuity and charge neutrality equations for all temperatures and generation rates. The procedure could have been implemented by numerically solving the integrals describing the trapped charge and the recombination rates, but the computing time was minimized by using approximate analytic solutions for those integrals. Our approach is based on considering that transport, trapping and recombination of carriers take place via a multiple-trapping (MT) process. In this MT model free carriers move by continuous trapping and release into and from localized states. However, the possibility of carrier hopping through localized states should also be considered, as suggested by some experimental results $[4,16,17]$. According to these works, hopping may become the dominant transport mechanism at low temperatures (low free carrier densities), especially in samples with a high defect density. The fundamental equations describing hopping conductivity are presented in 
appendix A. In the present work, we perform steady-state photoconductivity measurements under a quite intense illumination. Taking into account that the photoconductivity of a-Si:H is about five orders of magnitude larger than dark conductivity (for room temperature and below), the large number of charge carriers excited by the light to the bands, where the extended states are located, certainly implies that the transport channel through extended states dominates over transport by hopping between localized states. Indeed, Merazga et al. [18] reproduce with a numerical simulation the photoconductivity measurements over a wide range of temperatures (25-500 K) for device-quality a-Si:H samples of different doping levels. In their numerical simulations, carriers' transitions occur from localized states to extended states and vice versa (multiple-trapping process) and also from localized to localized states (hopping process).They conclude that, for undoped device-quality a-Si:H at a generation rates of $10^{20} \mathrm{~cm}^{-3} \mathrm{~s}^{-1}$, the hopping mechanism is predominant only for temperatures below $110 \mathrm{~K}$, being the multiple trapping mechanism the one that dominates for higher temperatures (see Fig. 7 in Ref. [18]). Similar results are obtained by Longeaud and Tobbeche in Ref. [19]. Based on these results we have neglected hopping in our equations, since our steady-state photoconductivity measurements are performed at similar generation rates and temperatures larger than $130 \mathrm{~K}$.

This paper is organized as follows. In section 2 we present some preliminary considerations about the DOS of a-Si:H and the basics of the method. In section 3 we test the proposed method through a numerical simulation for undoped a-Si: $\mathrm{H}$. In section 4 we describe the experimental procedures that we apply to find the material parameters of an a-Si:H sample. In this case, the ambipolar diffusion length was measured with the steady state photocarrier grating (SSPG) technique [20]. In section 5 we present and discuss the results. We compare the value that we obtain for the slope of the valence band tail with the Urbach tail of the absorption coefficient measured from transmittance/reflectance and Fourier transform photocurrent spectroscopy [21]. We also compare the part of the DOS corresponding to the conduction band tail and the deep states with the results obtained from the high frequency modulated photocurrent (MPC) experiment [22]. Finally, the conclusions of the

previous sections are summarized in section 6 . Three appendices are added to present a compendium of basic definitions and elemental equations, and to detail some of the approximations used in this work.

\section{Basics of the method}

Figure 1 shows the density of states of undoped a-Si:H that we will use as a basis for our discussion. This DOS can be considered as typical for device-grade a-Si:H, according to the current knowledge of the material [4]. The conduction band tail (CBT) is usually taken as acceptor-like, while the valence band tail (VBT) is generally taken as donor-like. These localized monovalent states (i.e., that can only be singly occupied) are represented by exponential functions decreasing from the band edges into the gap, and are a consequence of the long range disorder of the amorphous atomic lattice. The parameters that describe the decrease of the exponential functions are the characteristic temperatures $T_{C}$ for the CBT and $T_{V}$ for the VBT. Conversely, the deep states are amphoteric or bivalent in nature, i.e., they can be doubly occupied by electrons or holes, and for a-Si:H they have got a positive correlation energy $U$ greater than $0.1 \mathrm{eV}$ [23]. Occupying a bivalent state with one electron requires an energy $E$, while occupying the same state with a second electron requires a greater energy $E+U$. These states are a consequence of coordination defects in the lattice, especially dangling bonds (DB) [4]. The functional 
dependence with energy of these states when occupied with one electron is generally described by several Gaussian functions distributed along the mobility gap. Each Gaussian function is characterized by the integrated area $N_{D B}^{i}$, the energetic position $E_{D B}^{i}$ and the standard deviation $\omega_{i}$. According to the previous description, the DOS in the mobility gap of a-Si:H as a function of the first electron energy is:

$$
N(E)=N\left(E_{V}\right) e^{\frac{E_{V}-E}{k_{b} T_{V}}}+N\left(E_{C}\right) e^{\frac{E-E_{C}}{k_{b} T_{C}}}+\sum_{i} \frac{N_{D B}^{i}}{\sqrt{2 \pi} \omega_{i}} e^{\frac{-\left(E-E_{D B}^{i}\right)^{2}}{2 \omega_{i}{ }^{2}}}
$$

where the first term corresponds to the valence band tail $N_{V B T}(E)$, the second to the conduction band tail $N_{C B T}(E)$ and the third term $N_{D B}(E)$ is the summation over $i$ Gaussian functions representing the deep states. $k_{b}$ is Boltzmann's constant.

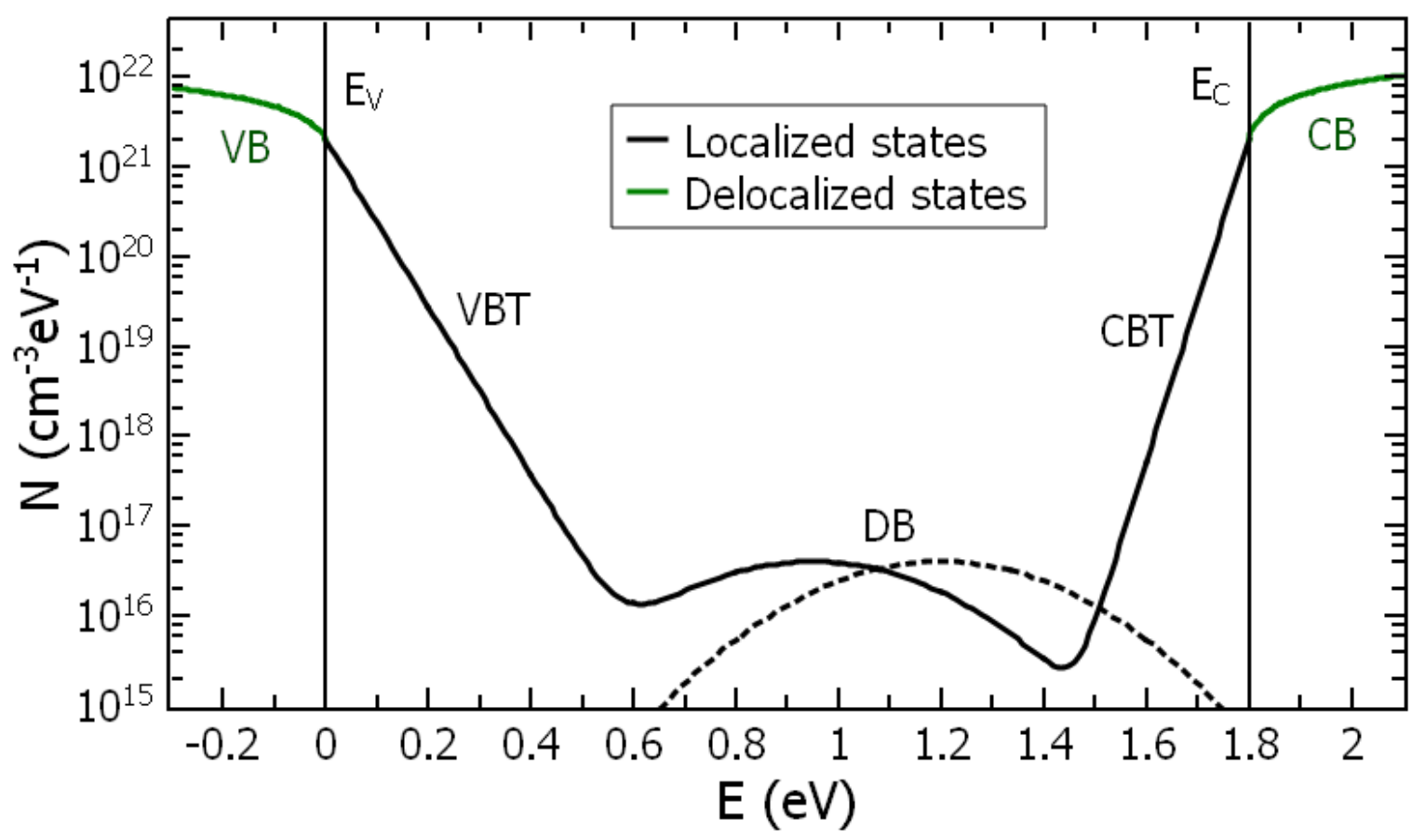

Figure 1. The continuous line corresponds to the undoped a-Si:H density of states used in the numerical simulations, with the dangling bonds states plotted as if they were singly occupied. The dash line shows the DB density of states while they are doubly occupied.

A localized monovalent acceptor state is neutral when occupied with a hole, and in this case can trap an electron (but not another hole). When occupied with an electron it is negatively charged and can trap a hole. As a consequence, an acceptor state is usually designated with the 0/- symbol, which represents its possible charge states. On the other hand, a monovalent donor state is positively charged when filled with a hole and has no charge when occupied with an electron (therefore, it is represented with the $+/ 0$ symbol). As a consequence of the electrostatic interaction between the trap and the free carriers, for acceptor states the hole capture coefficient $\left(c_{p}\right)$ should be larger than the electron capture coefficient $\left(c_{n}\right)$. On the other hand, for donor states the opposite inequality should hold $\left(c_{n}>c_{p}\right)$ [24]. 
A bivalent state has three different charge possibilities (+/0/-): it is positive when occupied with two holes, neutral when occupied with one hole and one electron, and negative when filled with two electrons. Consequently, these states have four capture coefficients: the positive ones have a unique electronic capture coefficient $\left(c_{n}^{+}\right)$because they cannot trap holes; the neutral states have an electron $\left(c_{n}^{0}\right)$ and a hole $\left(c_{p}^{0}\right)$ capture coefficient; and the negative ones have a unique hole capture coefficient $\left(c_{p}^{-}\right)$because they cannot trap electrons. According to the Coulombian interaction between the amphoteric state and the free carriers, the following inequalities must be verified: $c_{n}^{+}, c_{p}^{-}>c_{n}^{0}, c_{p}^{0}$ [24]. The steady-state occupation functions of monovalent and amphoteric states are presented in appendix A.

For an amorphous semiconductor in steady state at a given absolute temperature $T$ and under a certain uniform generation rate $G$, the system of equations composed by charge neutrality and continuity equations is verified. The charge neutrality equation can be written as

$$
Q^{+}-Q^{-}=0
$$

where $Q^{+}$is the total positive charge and $Q^{-}$the total negative charge. Each of them is the sum of three terms,

$$
Q^{+}=p+p^{\mathrm{VBT}}+p^{\mathrm{DB}} ; \quad Q^{-}=n+n^{\mathrm{CBT}}+n^{\mathrm{DB}} .
$$

$p, p^{V B T}$ and $p^{D B}$ are the concentrations of free holes, trapped holes in the valence band tail and trapped holes in the dangling bonds, respectively. Similarly, $n, n^{C B T}$ and $n^{D B}$ represent the free electron concentration, those trapped in the conduction band tail and those trapped in the dangling bonds, respectively. The steady-state continuity equation is

$$
G-R=0,
$$

where $R$ is the recombination rate and can be written

$$
R=\left(n p-N_{C} N_{V} e^{\frac{E_{V}-E_{C}}{k_{b} T}}\right)\left(R^{C B T}+R^{V B T}+R^{D B}\right) .
$$

$N_{C}$ and $N_{V}$ correspond to the effective DOS in the conduction and valence band, respectively, and their general definitions are presented in equations (A3) and (A4). $R^{C B T}, R^{V B T}$ and $R^{D B}$ involve the recombination terms though the conduction band tail, the valence band tail, and the deep states, respectively. Similarly to $p^{V B T}, p^{D B}, n^{C B T}$ and $n^{D B}$, these parameters correspond to an integral between $E_{V}$ and $E_{C}$, where the integrand is proportional to the region of the DOS mentioned in the superindex. Their precise definitions are presented in appendix $B$.

The shape of the delocalized DOS, as well as the temperature, defines the value of the effective DOS at the bands. In a-Si:H the energy distance between the Fermi level and the mobility edges is never less than $0.15 \mathrm{eV}$ [4], therefore the ones in the denominator of equations (A3) and (A4) can be discarded. If we also assume a square root energy dependence of the conduction and valence bands, as in crystalline silicon (see figure 1), we obtain the following expression for the effective delocalized DOS $[25,26]$ : 


$$
N_{l}=N\left(E_{l}\right) k_{b} T \sqrt{\frac{\pi T}{2 T_{l}}} e^{\frac{T_{l}}{2 T}}\left(1-\operatorname{erf}\left(\sqrt{\frac{T_{l}}{2 T}}\right)+\sqrt{\frac{2 T_{l}}{\pi T}} e^{\frac{-T_{l}}{2 T}}\right), l=C, V .
$$

For undoped a-Si:H (and also for n-type doped) $\mu_{n} n \gg \mu_{p} p$, where $\mu_{n}$ and $\mu_{p}$ are the free electron and hole mobility, respectively. In this case, it can be shown from equations (A5) and (A7) (and for typical values of the parameters involved) that the following expressions allow obtaining the steadystate free carrier concentrations within $1 \%$ error:

$$
\begin{aligned}
& n \cong \frac{1}{\mu_{n}}\left(\frac{\sigma}{q}-\frac{G L_{a m b}^{2}}{2 k_{b} T}\right), \\
& p \cong \frac{G L_{a m b}^{2} \sigma}{\mu_{p}\left(2 k_{b} T \sigma-q G L_{a m b}^{2}\right)},
\end{aligned}
$$

where $q$ is the absolute value of the electron charge, $\sigma$ the steady state conductivity under illumination and $L_{a m b}$ the ambipolar diffusion length. These equations assume that, in equation (A7), the thermal equilibrium concentrations of free carriers are much lower than the concentrations of photogenerated carriers, i.e., they are valid while the temperature is not extremely high nor the generation rate is extremely low. As a consequence of equations (7) and (8), the pair of equations (2) and (4) are functions of two parameters that can be measured directly, $\sigma(T, G)$ and $L_{a m b}(T, G)$, and the rest of the material parameters: the free carrier mobilities, the capture coefficients and the parameters associated to the DOS shape.

Initially, we have $m$ pairs of $\sigma$ and $L_{a m b}$ values for given $T$ and $G$ values. In order to test how well a randomly chosen set of parameters satisfies simultaneously equations (2) and (4), we define the following norm function:

$$
N=\sum_{i=1}^{m}\left[\left(2 \frac{Q_{i}^{+}-Q_{i}^{-}}{Q_{i}^{+}+Q_{i}^{-}}\right)^{2}+\left(\frac{G_{i}-R_{i}}{G_{i}}\right)^{2}\right] .
$$

Equation (9) is the sum of the squared relative errors between the two terms that constitute equations (2) and (4), for every pair of measured values $\sigma(T, G)$ and $L_{a m b}(T, G)$. In the second term between the square brackets of equation (9) we divide only by $G$ because it does not depend on the choice of the material parameters. With the correct material parameters, $N$ should theoretically be zero. As a consequence of experimental errors $N$ will never be strictly zero, but we assume that it will be smaller as the material parameters get closer to the correct ones.

In order to implement the TLBO algorithm, we define as in Ref. [14] a vector $\boldsymbol{X}$ whose components correspond to the material parameters we want to find. We generate a population of vectors $\boldsymbol{X}_{k}$, with values chosen randomly within a range of allowed values previously defined for each parameter. Then, we use the norm function (9) to evaluate which group of material parameters is the best, i.e., which one minimizes the norm function. This specific set of parameters corresponds to the teacher vector, $\boldsymbol{X}_{t}$, upon which we modify the rest of the population using the expression

$$
\boldsymbol{X}_{k}^{\prime}=\boldsymbol{X}_{k}+s\left(\boldsymbol{X}_{t}-\boldsymbol{X}_{k}\right), k \neq t,
$$


where $s$ is a positive number lower than 1 . Then we calculate the norm for each $\boldsymbol{X}_{k}{ }_{k}$ and from the lowest norm we define a new teacher vector $\boldsymbol{X}_{t}$, upon which we modify again the population. This process continues until the whole vector population is sufficiently close to the teacher vector, and in this way we find the set of parameters that best represents the material. The convergence is faster when $s$ is closer to one, but the parameter's space is better explored when $s$ is closer to zero.

\section{Numerical simulations}

Table 1. The first two columns contain the parameters names and the values used as input in the numerical simulation, corresponding to undoped a-Si:H. The third column presents the parameters obtained as a result of the application of the TLBO algorithm.

\begin{tabular}{lll}
\hline Parameter & Initial Value & TLBO \\
\hline$r^{C B T}($ dimensionless $)$ & 0.5 & 0.49 \\
$r^{V B T}$ (dimensionless) & 2 & 3.1 \\
$r^{+}$(dimensionless) & 4 & 4.6 \\
$r$ (dimensionless) & 0.25 & 0.24 \\
$c_{n}^{C B T}\left(\mathrm{~cm}^{3} \mathrm{~s}^{-1}\right)$ & $3 \times 10^{-8}$ & $3.8 \times 10^{-8}$ \\
$c_{n}^{V B T}\left(\mathrm{~cm}^{3} \mathrm{~s}^{-1}\right)$ & $4 \times 10^{-9}$ & $5.7 \times 10^{-9}$ \\
$c_{n}^{0}\left(\mathrm{~cm}^{3} \mathrm{~s}^{-1}\right)$ & $1 \times 10^{-8}$ & $1.4 \times 10^{-8}$ \\
$c_{n}^{+}\left(\mathrm{cm}^{3} \mathrm{~s}^{-1}\right)$ & $4 \times 10^{-8}$ & $7.3 \times 10^{-8}$ \\
$\mu_{n}\left(\mathrm{~cm}^{2} \mathrm{~V}^{-1} \mathrm{~s}^{-1}\right)$ & 18 & 20 \\
$\mu_{p}\left(\mathrm{~cm}^{2} \mathrm{~V}^{-1} \mathrm{~s}^{-1}\right)$ & 2 & 2.4 \\
$N\left(E_{V}\right)\left(\mathrm{cm}^{-3} \mathrm{eV}^{-1}\right)$ & $2 \times 10^{21}$ & $2.9 \times 10^{21}$ \\
$N\left(E_{C}\right)\left(\mathrm{cm}^{-3} \mathrm{eV}^{-1}\right)$ & $2 \times 10^{21}$ & $2.2 \times 10^{21}$ \\
$\left.N_{D B}^{1}(\mathrm{~cm})^{-3}\right)$ & $2 \times 10^{16}$ & $1.7 \times 10^{16}$ \\
$\omega_{1}(\mathrm{eV})$ & 0.2 & 0.26 \\
$U(\mathrm{eV})$ & 0.25 & 0.24 \\
$E_{D B}^{1}(\mathrm{eV})$ & 0.95 & 0.93 \\
$E_{C}(\mathrm{eV})$ & 1.8 & 1.79 \\
$T_{C}(\mathrm{~K})$ & 280 & 274 \\
$T_{V}(\mathrm{~K})$ & 540 & 534 \\
\hline
\end{tabular}

In this section we test through numerical simulations the ability of the method that we have proposed to obtain the material parameters of a-Si:H. We follow the procedure that would be implemented experimentally, first obtaining the steady-state conductivity under illumination and the ambipolar diffusion length for different temperatures and generation rates (equations (A5) and (A7)). For that purpose, using typical material parameters for undoped a-Si:H (listed in the first two columns of table 1), the fundamental equations of section 2 and appendix A are solved without approximations. By solving the charge neutrality equation for a certain temperature under dark conditions we find the Fermi level, and with this value we obtain the thermal equilibrium free carrier concentrations, $n_{e}$ and $p_{e}$ [27]. For the same temperature and different generation rates, we calculate the steady-state free carrier concentrations $n$ and $p$ by solving simultaneously the continuity and charge neutrality equations. The integrals are solved numerically without approximation, using the trapezoidal rule after discretization of 
the energy interval. For simplicity, from now on we use just one Gaussian function to describe the deep states, and we take the valence band edge as the energy reference $\left(E_{V}=0\right)$, so that $E_{C}$ is coincident with the mobility-gap energy.

We numerically obtain a total of 25 pairs of values $\left(\sigma, L_{a m b}\right)$, for 5 different temperatures between 130 and $290 \mathrm{~K}$ separated by $40 \mathrm{~K}$, and 5 different generation rates for each temperature from $5.2 \times 10^{18}$ to $4.2 \times 10^{20} \mathrm{~cm}^{-3} \mathrm{~s}^{-1}$, obtained by dividing successively by 3 the highest generation rate value. These values are treated as if they were measurements, applying to them the TLBO algorithm to retrieve the set of parameters that better satisfies the approximate analytic solutions to the continuity and charge neutrality equations obtained in appendices $B$ and $C$.

The initial ranges of allowed values for the different material parameters, needed to implement the algorithm, are listed in table 2. Generally, we used initial populations of 100000 vectors and $s$ values near 0.13 . The procedure consists in running the algorithm with the initial intervals dozens of times until convergence. Then we compare the different material parameters obtained in each run, and if we observe that some parameters vary on a narrower interval we reduce the initially assigned interval. We continue with this intervals reduction until no smaller value of the norm is obtained. The whole procedure takes several hours on a desktop computer.

Table 2. Initial ranges of allowed values for the material parameters corresponding to undoped a-Si:H. They were taken from the specific literature of the material [4] and the theoretical inequalities mentioned in section 2 .

\begin{tabular}{cc}
\hline Parameter & Initial Range \\
\hline$N\left(E_{C}\right), N\left(E_{V}\right)\left(\mathrm{cm}^{-3} \mathrm{eV}^{-1}\right)$ & {$\left[9 \times 10^{20}, 8 \times 10^{21}\right]$} \\
$r^{V B T}, r^{+}($dimensionless $)$ & {$[1,25]$} \\
$r^{C B T}, r^{-}($dimensionless $)$ & {$[0.04,1]$} \\
$c_{n}^{C B T}, c_{n}^{V B T}, c_{n}^{0}, c_{n}^{+}\left(\mathrm{cm}^{3} \mathrm{~s}^{-1}\right)$ & {$\left[10^{-9}, 4 \times 10^{-7}\right]$} \\
$\mu_{n}\left(\mathrm{~cm}^{2} \mathrm{~V}^{-1} \mathrm{~s}^{-1}\right)$ & {$[8,40]$} \\
$\mu_{p}\left(\mathrm{~cm}^{2} V^{-1} s^{-1}\right)$ & {$[0.4,5]$} \\
$E_{C}(\mathrm{eV})$ & {$[1.7,1.9]$} \\
$T_{C}(\mathrm{~K})$ & {$[220,380]$} \\
$T_{V}(\mathrm{~K})$ & {$[450,680]$} \\
$U(\mathrm{eV})$ & {$[0.1,0.5]$} \\
$\omega_{1}(\mathrm{eV})$ & {$[0.04,0.9]$} \\
$E_{D B}^{1}(\mathrm{eV})$ & {$[0.4,1.35]$} \\
$N_{D B}^{1}(\mathrm{~cm}-3)$ & {$\left[5 \times 10^{15}, 6 \times 10^{17}\right]$} \\
\hline
\end{tabular}

In the third column of table 1 we present the results obtained when the TLBO algorithm is used to minimize the norm function defined by equation (9). Without approximations, these values should be identical to the initially introduced ones. However, the approximations introduced in appendices $B$ and $C$ imply that they differ. It can be seen that the largest error is obtained for $c_{n}^{+}(83 \%)$. The error in the rest of the parameters is not higher than $55 \%$, consequently we can conclude that the procedure allows estimating all the transport material parameters. The largest errors are found in those parameters whose initial intervals are also the largest, i.e., in capture coefficients. Besides, we have tested other 
norm functions that take into account separately the charge neutrality and the continuity equations. In these tests, the errors obtained for the material parameters were generally larger. Moreover, the capture coefficients cannot be obtained individually if the charge neutrality equation is used alone, as this equation depends only on the ratios between capture coefficients (see appendix B).

Other DOS shapes have been tested, obtaining in all cases similar results: the largest errors are in some capture coefficients, while the mobilities and parameters describing the DOS shape are well reproduced. In figure 2 we have plotted the localized one-electron effective density of states $N_{\text {eff, }}$ which is equal to the DOS given by equation (1) plus the DB states when they are doubly occupied:

$$
N_{e f f}(E)=N(E)+\frac{N_{D B}^{1}}{\sqrt{2 \pi} \omega_{1}} e^{\frac{-\left(E-E_{D B}^{1}-U\right)^{2}}{2 \omega_{1}^{2}}} .
$$

Observe that $N_{\text {eff }}$ is also a function of the correlation energy. The continuous curve in figure 2 corresponds to the initially introduced DOS (second column of table 1), while the dashed curve was obtained with the parameters given by the optimization algorithm (third column of table 1). A very good agreement between both curves can be observed, suggesting that the proposed method is especially useful for the DOS determination.

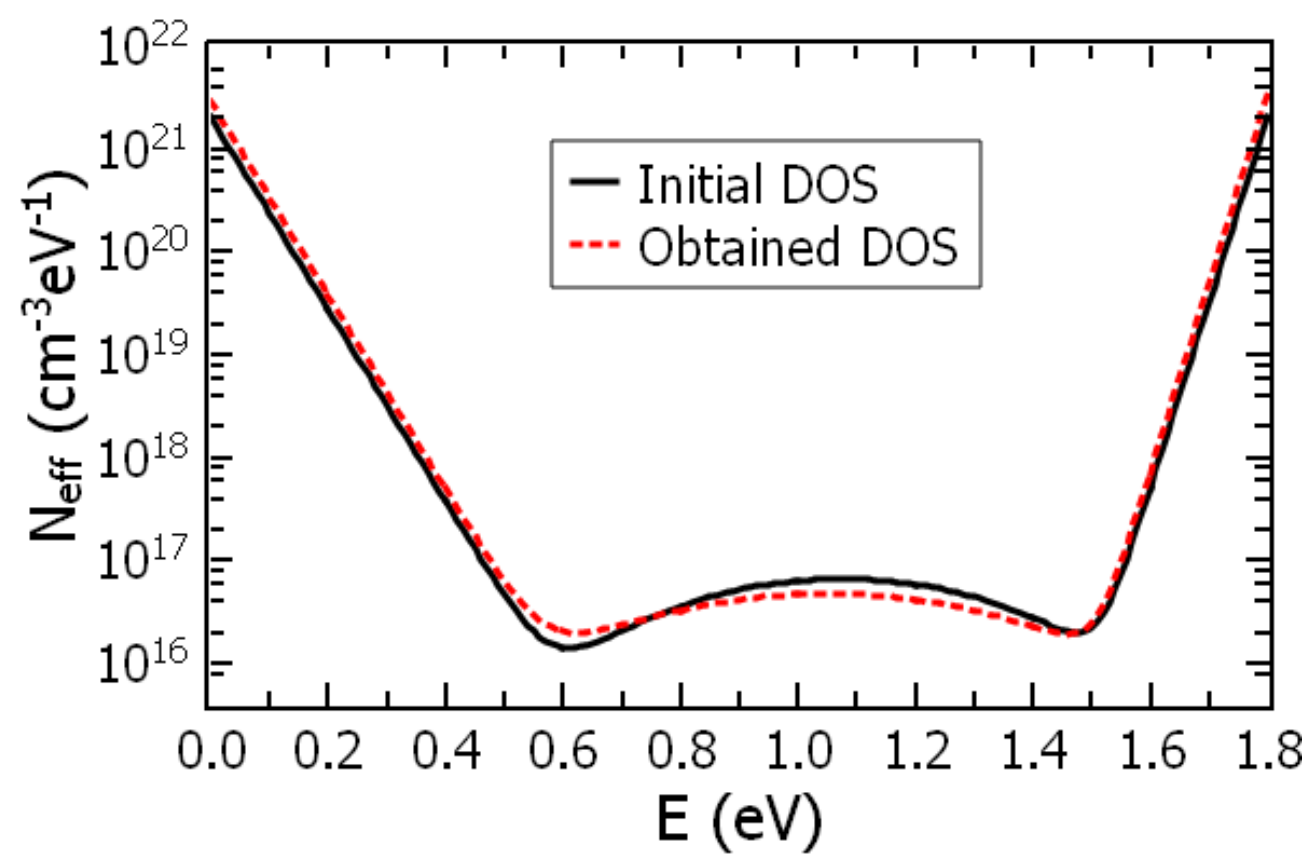

Figure 2. Localized one-electron effective density of states as a function of energy. The red dashed line corresponds to the DOS obtained after application of the TLBO algorithm, while the black solid line was obtained with the initial parameters (second column of table 1).

Even when using the exact material parameters, the norm given by equation (9) is not zero due to the analytical approximations introduced in appendix B. The value of the norm function, obtained when using the exact material parameters (second column of table 1 ), is $7.32 \times 10^{-3}$. After application of 
the TLBO algorithm, the norm value decreases to $6.74 \times 10^{-4}$. This smaller value indicates than the TLBO algorithm works properly, partially correcting the errors coming from the analytical approximations.

\section{Experimental methods}

Having tested numerically the applicability of the method, we have applied it to a device-grade aSi:H sample. It was deposited on a Corning 1737 glass substrate by Plasma Enhanced Chemical Vapor Deposition (PECVD), in a conventional capacitively coupled reactor operated at $50 \mathrm{MHz}$, from pure silane at a flow rate of $40 \mathrm{sccm}$, a chamber pressure of 0.53 Torr and a substrate temperature of $250{ }^{\circ} \mathrm{C}$. Two parallel silver paint electrodes were deposited on top of the sample for electrical contacts, with a height of $1 \mathrm{~cm}$ and a separation of $1 \mathrm{~mm}$ between them. After a proper annealing, the ohmicity of the contacts was checked. The sample was light-soaked during 2 hours with a He-Ne laser $\left(100 \mathrm{~mW} / \mathrm{cm}^{2}\right)$ to minimize the effects of light-induced changes on the transport parameters during the experiments [28]. All the measurements were performed in a cryostat evacuated to a pressure lower than $10^{-6}$ Torr, to avoid the presence of adsorbed gases.

The film thickness was measured by making use of the interference pattern of the UV- vis (400-1750 $\mathrm{nm}$ ) transmittance and reflectance spectra, obtaining a value of $L=0.49 \mu \mathrm{m}$. The absorption coefficient $\alpha$ in the strong absorption region was also evaluated at room temperature ( $298 \mathrm{~K})$ from the UV-vis spectra, while the subgap $\alpha$ was measured by means of the Fourier-Transform Photocurrent Spectroscopy (FTPS) technique [21].

The SSPG and steady state conductivity measurements have been made using a He-Ne laser (633 nm or $1.96 \mathrm{eV}$ ), for light fluxes $\Phi$ between $10^{15}$ and $5 \times 10^{16} \mathrm{~s}^{-1} \mathrm{~cm}^{-2}$, and for the following temperatures: 130, $150,170,210,230,270$ and $290 \mathrm{~K}$. The description of the automated equipment used for these measurements can be found in Ref. [29]. The SSPG technique consists on measuring the electrical current that results when the sample is illuminated with two beams of different intensities that strike the sample forming an angle. When the beams are coherent and have the same polarization parallel to the electrodes, a sinusoidal interference pattern is created in the direction of the current between the electrical contacts, while the illumination is uniform when the beams have perpendicular polarizations. It is necessary to perform measurements of the photocurrent with and without the interference pattern to obtain the ratio between them, $\beta$. Finally, a fit of $\beta$ as a function of the interference grating period $\Lambda$ is made using the Ritter-Zeldov-Weiser (RZW) equation [20]:

$$
\beta=1-\frac{2 \varphi}{\left(1+\left(\frac{2 \pi L_{a m b}}{\Lambda}\right)^{2}\right)^{2}},
$$

where $\varphi$ and $L_{a m b}$ are fit parameters. We use the RZW equation to obtain $L_{a m b}$ for its simplicity, although other formulas have also been proposed in the literature [30]. The determination of the best formula to extract $L_{a m b}$ from SSPG measurements is beyond the scope of the present work.

In figure 3 we present the experimental results measured on the sample, as a function of the generation rate and for different temperatures. Figure 3a) is the steady-state conductivity under illumination, and figure $3 \mathrm{~b}$ ) the ambipolar diffusion length obtained from SSPG measurements. Figure $3 b$ ) includes the statistical errors obtained for $L_{a m b}$ when the SSPG experimental data are fitted with equation (12). When the relative error is calculated, the trend is clear: the error increases when the 
generation rate increases and when the temperature decreases. The increment of the relative error is a consequence of the decrease of $L_{a m b}$ with higher generation rates and smaller temperatures, as can be seen in figure $3 b$ ). With the exception of equation (B11), the approximations made in this work are generally better for smaller temperatures and higher generation rates, although the $L_{a m b}$ values obtained from SSPG are less precise in this case. As a consequence, for low temperatures we only performed measurements for small generation rates (see figure $3 \mathrm{~b}$ ).
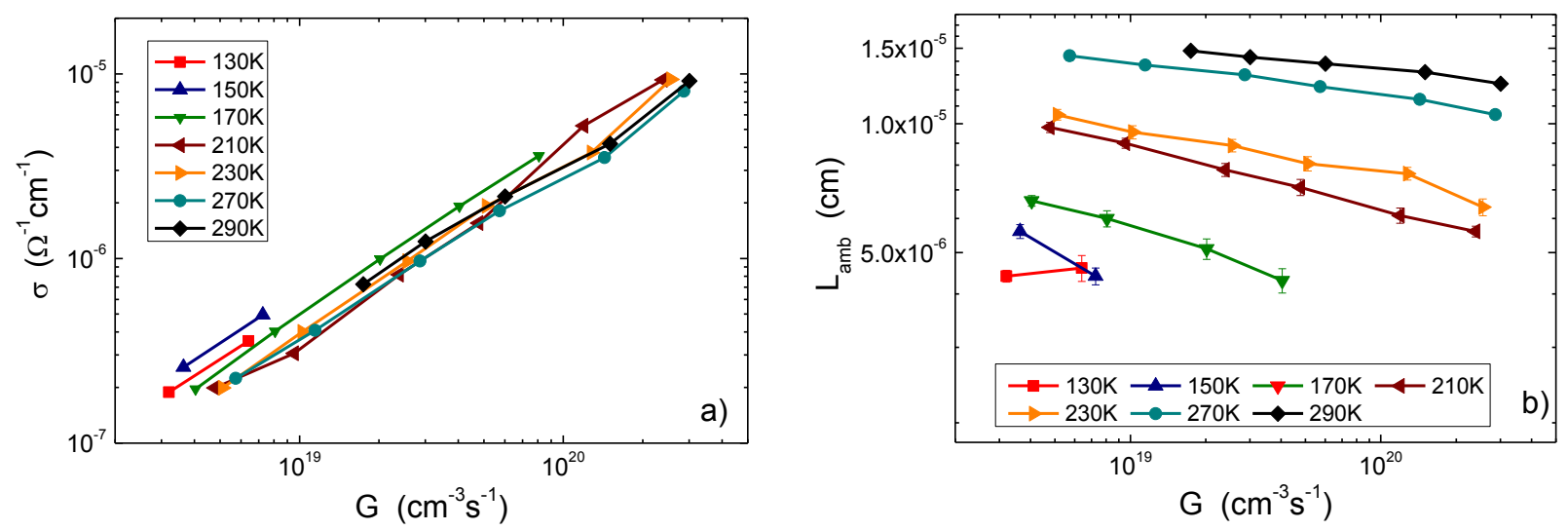

Figure 3. Experimental results of steady-state conductivity under illumination (a) and ambipolar diffusion length measured by SSPG (b), as a function of generation rate, for different temperatures indicated in the inset. The error bars in (b) (lower than the symbol size in some cases) correspond to the statistical errors obtained from a fit with equation (12) of the SSPG data.

The modulated photocurrent experiment was performed illuminating the sample with a red light $(625 \mathrm{~nm}$ or $1.98 \mathrm{eV})$ modulated at an angular frequency $\omega$, at temperatures from 110 to $330 \mathrm{~K}$ with a $20 \mathrm{~K}$ step. The high-frequency MPC allows obtaining an estimation of the DOS through the following equations [22]:

$$
\begin{aligned}
& \frac{c_{n}^{C B T} N^{C B T}\left(E_{\omega}\right)+c_{n}^{+} N^{D B}\left(E_{\omega}\right)+c_{n}^{0} N^{D B}\left(E_{\omega}-U\right)}{\mu_{n}}=\frac{2 q \xi G_{a c}|\sin (\phi)|}{\pi k_{b} T\left|J_{a c}\right|}, \\
& E_{\omega}=E_{C}-k_{b} T \ln \left(\frac{c_{n} k_{b} T N\left(E_{C}\right)}{\omega}\right) .
\end{aligned}
$$

It is necessary to measure the modulus $\left|J_{a c}\right|$ and the phase shift $\phi$ of the modulated photocurrent generated through a temporally modulated illumination superimposed to a uniform illumination of higher intensity, while a steady electric field $\xi$ is applied between the electrical contacts. The uniform and alternating light fluxes used were $10^{13}$ and $3 \times 10^{12} \mathrm{~cm}^{-2} \mathrm{~s}^{-1}$, respectively. $G_{a c}$ is the carrier generation rate corresponding to the modulated illumination. To get the energy scaling, in equation (14) we have to choose one of the three electronic capture coefficients obtained (see table 3 ). We used the $c_{n}^{0}$ value, because it is situated between $c_{n}^{+}$and $c_{n}^{C B T}$, although in figure 4 there was nearly no change to the naked eye when using any of the other two.

As usual, the mean generation rate for each light flux was obtained with the formula 


$$
G=\Phi \frac{(1-r)}{L}\left(1-e^{-\alpha L}\right)
$$

where $r$ is the reflection coefficient of the front surface. It can be found in the literature that $\alpha$ increases uniformly with temperature for the light energies $(\sim 1.97 \mathrm{eV})$ used in the experiments [31,32]. In Ref. [31] it can be seen that the value of $\alpha$ doubles when temperature changes from 151 to $293 \mathrm{~K}$. Not having found more precise measurements, we used this information to define a linear relation $\alpha(T)$. To implement the TLBO algorithm, we assigned the same initial intervals to the material parameters that were used in section 3 (table 2), and applied exactly the same procedure.

\section{Results and discussion}

Table 3 shows the material parameters obtained from the application of the TLBO algorithm to the measurements performed on the a-Si:H sample. The holes capture coefficients presented in table 3 are calculated from the electronic capture coefficients and the ratios between capture coefficients that were obtained. All the parameters are within the range of acceptable values for device-quality intrinsic a-Si:H. By requiring that $r^{V B T}, r^{+}>1$ and $r^{C B T}, r^{-}<1$ in the allowed initial intervals (see table 2), the inequalities $c_{n}^{V B T}>c_{p}^{V B T}, c_{p}^{C B T}>c_{n}^{C B T}, c_{n}^{+}>c_{p}^{0}$ and $c_{p}^{-}>c_{n}^{0}$ are guaranteed. Moreover, it can be observed in table 3 that the inequalities $c_{n}^{+}>c_{n}^{0}$ and $c_{p}^{-}>c_{p}^{0}$ are also fulfilled, as theoretically expected.

Table 3: Parameters obtained using the procedure described in section 2 for an undoped a-Si:H sample.

\begin{tabular}{cc}
\hline Parameter & Value \\
\hline$c_{n}^{C B T}$ & $9.0 \times 10^{-8} \mathrm{~cm}^{3} \mathrm{~s}^{-1}$ \\
$c_{p}^{C B T}$ & $1.2 \times 10^{-7} \mathrm{~cm}^{3} \mathrm{~s}^{-1}$ \\
$c_{n}^{V B T}$ & $3.0 \times 10^{-9} \mathrm{~cm}^{3} \mathrm{~s}^{-1}$ \\
$c_{p}^{V B T}$ & $5.0 \times 10^{-10} \mathrm{~cm}^{3} \mathrm{~s}^{-1}$ \\
$c_{n}^{0}$ & $1.2 \times 10^{-7} \mathrm{~cm}^{3} \mathrm{~s}^{-1}$ \\
$c_{p}^{-}$ & $6.9 \times 10^{-7} \mathrm{~cm}^{3} \mathrm{~s}^{-1}$ \\
$c_{n}^{+}$ & $3.9 \times 10^{-7} \mathrm{~cm}^{3} \mathrm{~s}^{-1}$ \\
$c_{p}^{0}$ & $2.5 \times 10^{-8} \mathrm{~cm}^{3} \mathrm{~s}^{-1}$ \\
$\mu_{n}$ & $37 \mathrm{~cm}^{2} \mathrm{~V}^{-1} \mathrm{~s}^{-1}$ \\
$\mu_{p}$ & $4.0 \mathrm{~cm}^{2} \mathrm{~V}^{-1} \mathrm{~s}^{-1}$ \\
$N\left(E_{V}\right)$ & $6.8 \times 10^{21} \mathrm{~cm}^{-3} \mathrm{eV}^{-1}$ \\
$N\left(E_{C}\right)$ & $6.4 \times 10^{21} \mathrm{~cm}^{-3} \mathrm{eV}^{-1}$ \\
$N_{D B}^{1}$ & $1.3 \times 10^{16} \mathrm{~cm}^{-3}$ \\
$\omega_{1}$ & $0.84 \mathrm{eV}$ \\
$U$ & $0.26 \mathrm{eV}$ \\
$E_{D B}^{1}$ & $0.96 \mathrm{eV}$ \\
$E_{C}$ & $1.75 \mathrm{eV}$ \\
$T_{C}$ & $257 \mathrm{~K}$ \\
$T_{V}$ & $531 \mathrm{~K}$ \\
\hline
\end{tabular}


Figure 4 (solid line) presents the one-electron effective DOS obtained from the application of our method, given by the parameters specified in table 3. In order to compare with the MPC measurements, the DOS is multiplied by the respective electronic capture coefficient and divided by the electron mobility, i.e., in the form of the left-hand side of equation (13) plus the corresponding VBT term. The deep states region is practically flat, as a consequence of the large value obtained for $\omega_{1}$ (see table 3). This shape of the DB density is consistent with the results of the improved Defect Pool Model (DPM) described in Ref. [7]. The green points in figure 4 were obtained applying equations (13) and (14) to the MPC measurements. The points plotted with the same symbol correspond to the same temperature (isotherm). For each temperature, the higher energy points correspond to the higher frequency measurements. As a result, it is expected that the solid line matches the high energy envelope generated by the isotherms (see equation (13)). As can be seen, the agreement between the results of our new method and the MPC experiment is quite good. As usual, the high frequency MPC envelope slightly overestimates the DOS in the energy region located between the CBT and the DB states [33,34]. However, a non-expected underestimation in the deep states region is also observed.

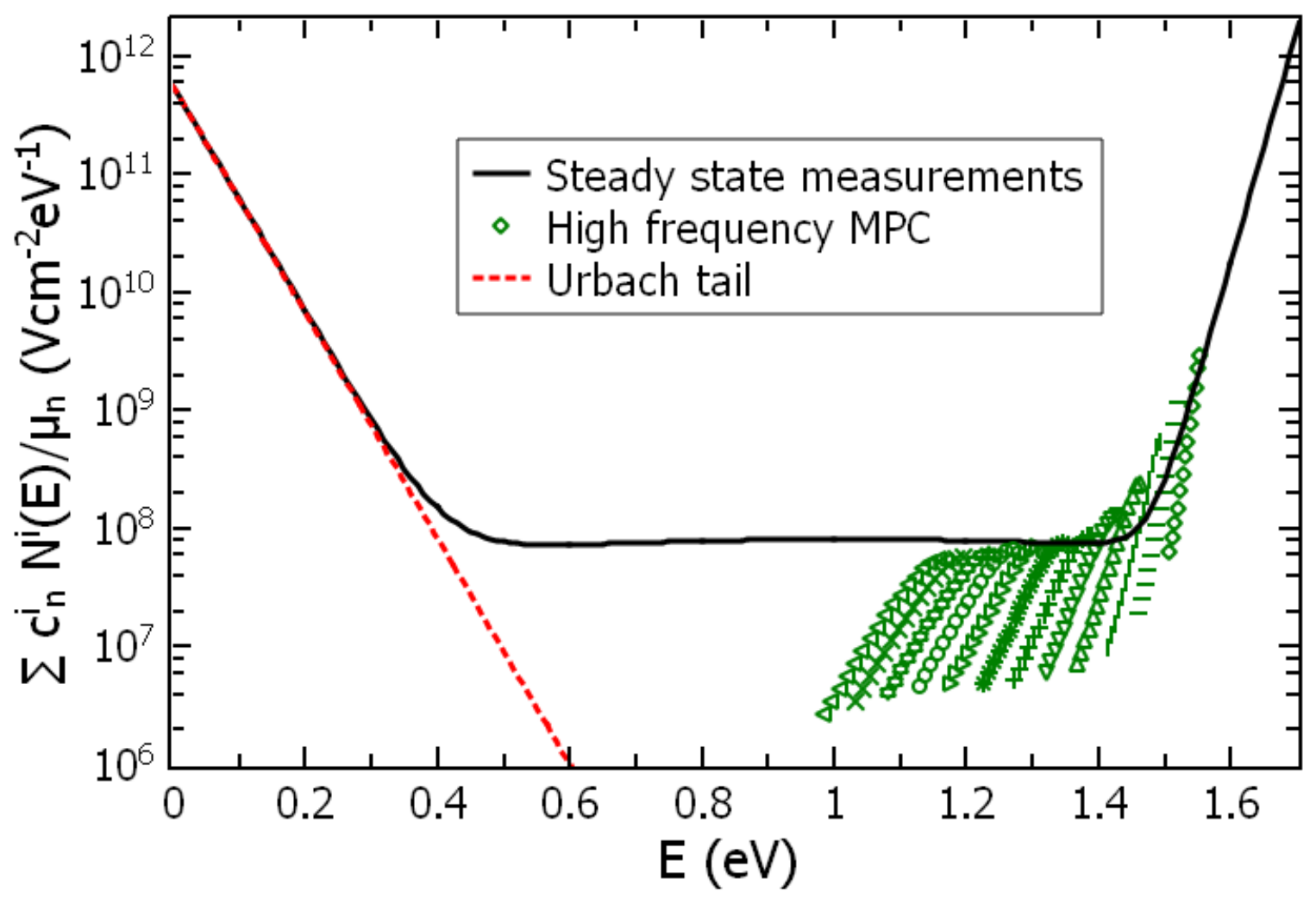

Figure 4. Density of localized states, multiplied by the respective electronic capture coefficient and divided by the electron mobility, as a function of energy. The black solid curve is the result of the TLBO optimization algorithm. The green points are MPC measurements, obtained applying equations (13) and (14) to the data, each symbol corresponding to a different temperature. The dashed red line corresponds to the VBT term obtained from FTPS measurements, corrected with Stutzmann's formula (see text). 
The decrease of the MPC curves in figure 4 for the higher temperatures (i.e., smaller energies), leading to a discrepancy with the solid line, may be a consequence of the temperature dependence of the deep states capture coefficients. As established from photoconductive time of flight (TOF) measurements $[35,36]$ the capture cross-sections of defect states decrease with the increase of temperature. For simplicity we make no difference between charged and neutral dangling bonds, although TOF experiments applied to doped and undoped samples suggest stronger temperature dependence for charged states. According to Ref. [36], for undoped samples the dangling bond capture cross-section $\sigma_{D B}$ goes approximately as $\sim T^{-1.5}$, and assuming that the thermal velocity $v_{t h}$ of the carriers has a dependence $\sim T^{0.5}$ [24], we finally get that $c_{n}^{0}, c_{n}^{+}=v_{t h} \sigma_{D B} \propto T^{-1}$. Figure 5 is a close-up of the MPC data region of figure 4, where we have also plotted with red dashed lines the deep states isotherms corrected to the median temperature of steady-state measurements $(230 \mathrm{~K})$. With this temperature correction, the agreement in the dangling bond region results excellent.

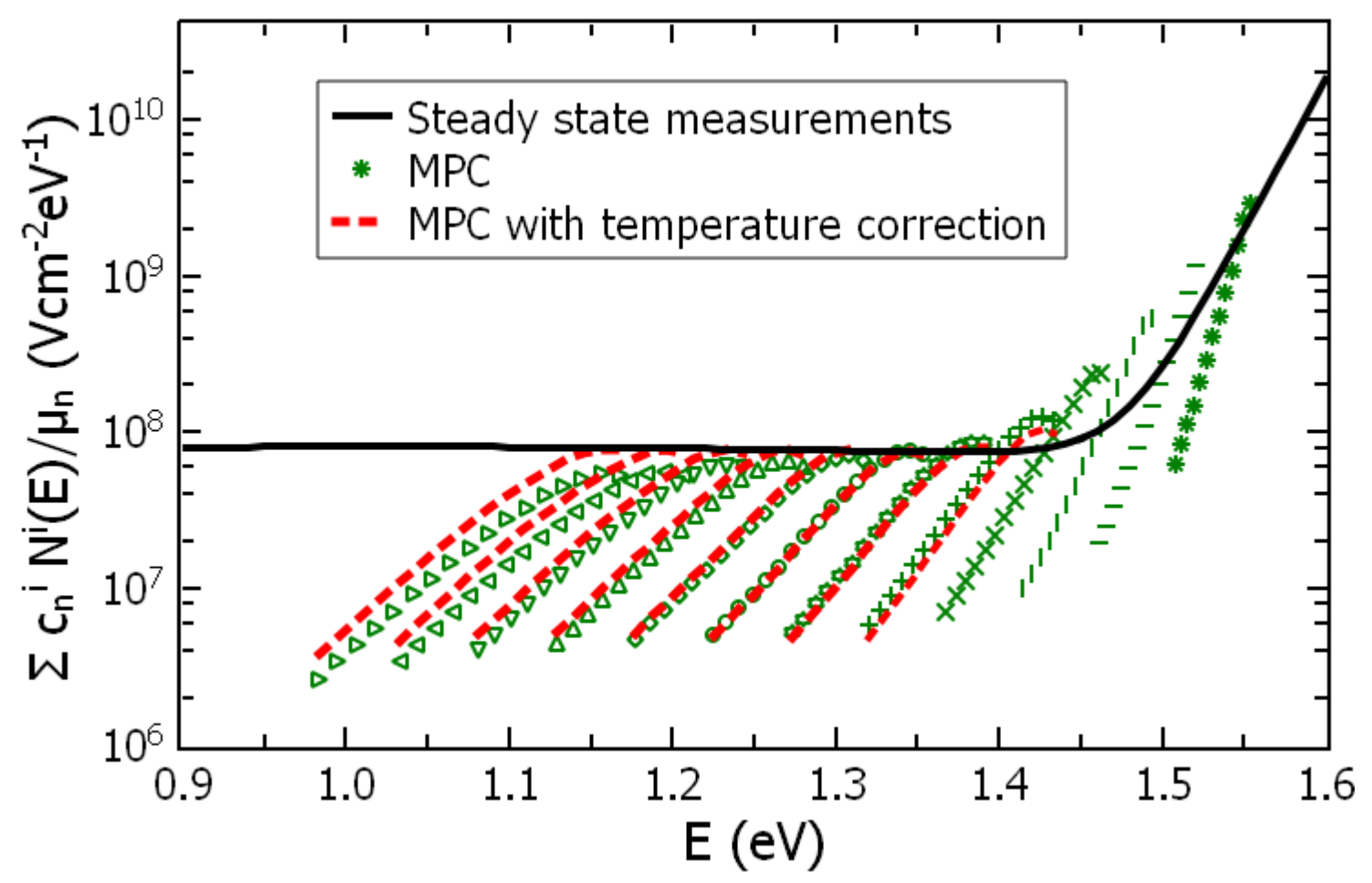

Figure 5. Density of localized states, multiplied by the respective electronic capture coefficient and divided by the electron mobility, as a function of energy. The black solid curve is obtained with the parameters of table 3 . The green points are obtained applying equations (13) and (14) to the MPC data. The red dashed lines correspond to the deep states MPC isotherms corrected to the median temperature $(230 \mathrm{~K})$ of steady-state measurements, assuming a $T^{1}$ dependence of the capture coefficients. 
Figure 6 presents the absorption coefficient vs. the incident photon energy measured as described in section 4 for $T \sim 295 \mathrm{~K}$, together with an exponential fit (note the logarithmic vertical scale) of the energy region known as Urbach tail [4]. In a-Si:H the CBT slope is much steeper than the VBT slope, so the slope of the Urbach tail reflects the VBT slope. Therefore, from the fit of figure 6 we get $T_{V}$ $=562 \mathrm{~K}$. Although for simplicity we assumed a temperature-independent DOS model, there are reports in the literature of a slight decrement in the VBT slope with temperature [37]. Stutzmann's formula with $\gamma=1$ gives the temperature-dependent expression $T_{V}(T)=\sqrt{478^{2}+T^{2}}$ for the VBT slope. The value obtained with the TLBO algorithm, $T_{V}=531 \mathrm{~K}$, is smaller than the one obtained from the Urbach tail measurement $\left(T_{V}=562 \mathrm{~K}\right)$, as expected considering that the Urbach tail was measured at a higher temperature. However, it is nearly equal to the value obtained with Stutzmann's formula for the median temperature of all measured values, $T=230 \mathrm{~K}$, i.e. $T_{V}(230 \mathrm{~K})=530 \mathrm{~K}$. This is indicative of an excellent agreement between both experimental techniques for the determination of the valence band tail slope (red dashed line in figure 4), and gives confidence to the method proposed in this work for the determination of $T_{V}$.

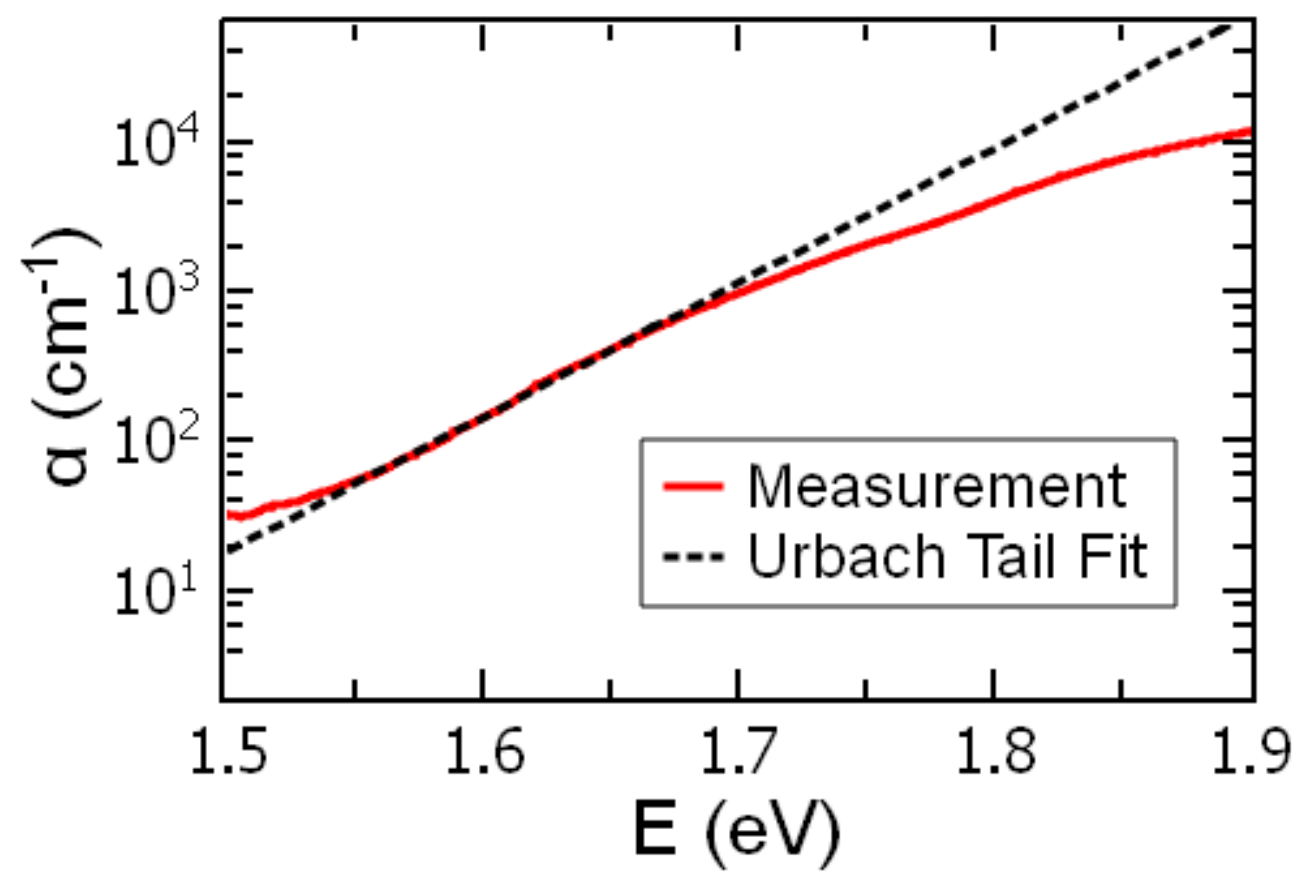

Figure 6. Absorption coefficient as a function of photon energy. The red curve corresponds to a measurement performed at $295 \mathrm{~K}$, while the black dashed line is the best fit to the Urbach tail region.

As a final test of the validity of the results, we have calculated the steady-state conductivity under illumination and the ambipolar diffusion length that result from the application of the transport equations with the parameters obtained from our method (table 3). Figure 7 presents the results, for the same temperatures and generation rates used in the experiments. Comparing with figure 3 , we see that the experimental results are very well reproduced, both for the conductivity and the diffusion 
length. Even the unusual behavior of the conductivity as a function of temperature of figure 3a), increasing from 130 to $150 \mathrm{~K}$ and then decreasing from 150 to $210 \mathrm{~K}$, is reproduced by the simulations in figure 7a). Regarding the diffusion length, all the features of figure $3 b$ ) are reproduced by figure $7 b$ ), except for the lowest temperature $(130 \mathrm{~K})$, where the increase of $L_{a m b}$ with $G$ found in figure $\left.3 \mathrm{~b}\right)$ is probably due to an experimental error. Therefore, we are quite confident on the capability of the proposed method to provide trustable mobilities and DOS estimations, and to offer good insight into the different capture coefficients.
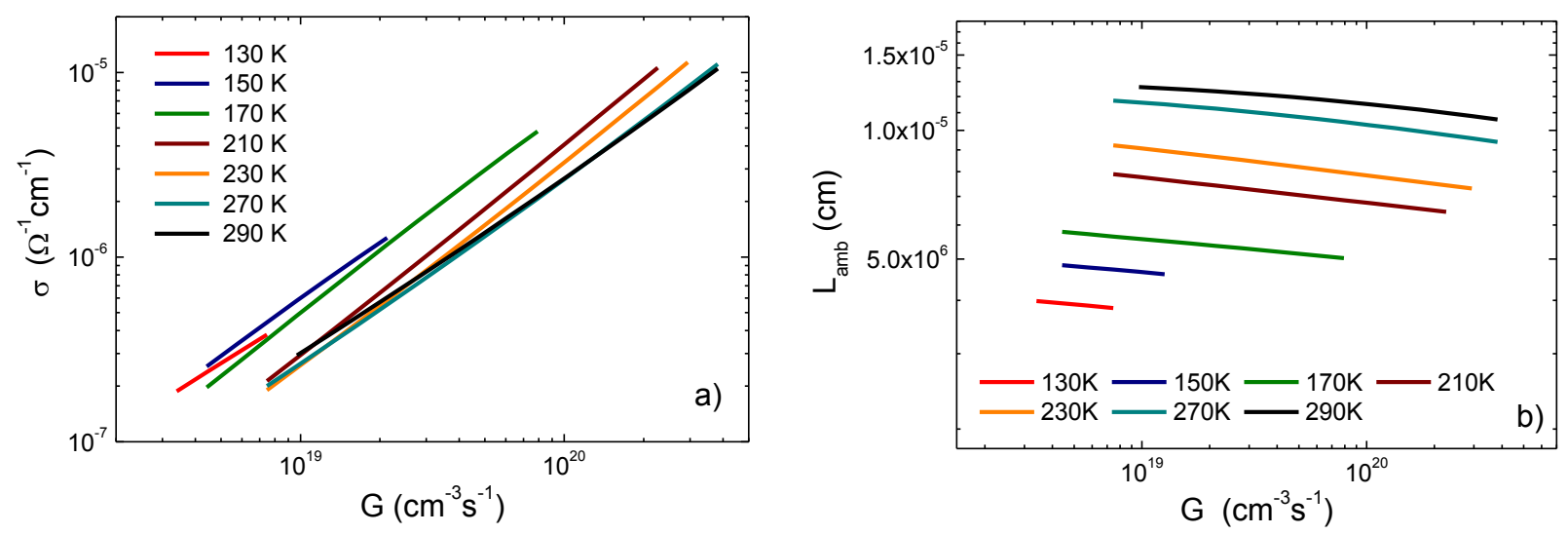

Figure 7. Calculated results of steady-state conductivity under illumination (a) and ambipolar diffusion length (b), as a function of generation rate, for different temperatures indicated in the inset. The calculations are performed solving the transport equations with the parameters of table 3.

Future investigations could explicitly include the temperature dependence of the VBT, the mobility gap and the capture coefficients $[4,24,35,36]$. Also, a better description of the deep states could be achieved by using several Gaussian functions [7], although these would notably increase the number of material transport parameters, making slower the convergence of the algorithm and increasing the computing time. The possibility of carrier hopping through localized states could also be included in the theoretical description. The method that we propose for intrinsic a-Si:H can be applied without change to $n$-doped a-Si:H samples, and could also be applied to $p$-doped samples after exchanging $n$ and $p$ in equations (7) and (8). Although there are several characterization methods for $\mathrm{a}-\mathrm{Si}: \mathrm{H}$ based on photoconductivity measurement for different temperatures and generation rates [11,38], we have not found in the specific literature a detailed measurement of the temperature dependence of its optical parameters at the light energy used to perform the experiments ( 1.97 eV). According to equation (15), the temperature dependence of the optical parameters is needed for a precise determination of the generation rate at different temperatures. The technique that we propose in this work, and others that also include the generation rate directly in their formulas, could be benefited from these measurements.

\section{Conclusion}

We proposed a method that allows obtaining all the transport parameters of a-Si:H from measurements of steady state photoconductivity and ambipolar diffusion length, performed at different 
temperatures and generation rates. The method consists in solving the continuity and charge neutrality equations using a simplified TLBO algorithm. In a numerical simulation we observed that the material parameters are obtained with less error when the pair of equations is solved simultaneously. In this case, the parameters exhibiting more uncertainty in their estimation are some of the capture coefficients, in agreement with the fact that these parameters also present the highest initial indeterminacy. With the mentioned theoretical analysis we conclude that the proposed methodology allows for a complete electrical characterization of undoped a-Si:H.

We also tested the procedure experimentally, applying it to an undoped a-Si:H sample. We compared the obtained $T_{V}$ value with the one measured from the Urbach tail and the agreement was excellent. The parameters corresponding to dangling bonds and conduction band tail were compared with high frequency MPC experiment, and the matching was also excellent. We are confident that the shape of the DOS can be obtained with good accuracy.

\section{Acknowledgments}

We acknowledge support from CONICET under Project Nos. PUE 22920160100054 CO and PIP 0414, ANPCyT under Project No. PICT 2013-2098, Universidad Nacional del Litoral under project CAI+D PI 84-099, and MINCYT - Ecos Sud under Project No. A13E02.

\section{Appendix A - Basic equations}

The equilibrium concentrations of free carriers under dark are given by

$$
\begin{aligned}
& n_{e}=\int_{E_{C}}^{+\infty} \frac{N(E) d E}{1+e^{\frac{E-E_{f}}{k_{b} T}}}=N_{C} e^{\frac{E_{f}-E_{C}}{k_{b} T}}, \\
& p_{e}=\int_{-\infty}^{E_{V}} \frac{N(E) d E}{1+e^{\frac{E_{f}-E}{k_{b} T}}}=N_{V} e^{\frac{E_{V}-E_{f}}{k_{b} T}},
\end{aligned}
$$

where $N_{C}$ and $N_{V}$ are the effective DOS at the band edges, defined as

$$
\begin{aligned}
& N_{C}=\exp \left(\frac{E_{C}-E_{f}}{k_{b} T}\right) \int_{E_{C}}^{+\infty} \frac{N(E) d E}{1+\exp \left(\frac{E-E_{f}}{k_{b} T}\right)^{\prime}}, \\
& N_{V}=\exp \left(\frac{E_{f}-E_{V}}{k_{b} T}\right) \int_{-\infty}^{E_{V}} \frac{N(E) d E}{1+\exp \left(\frac{E_{f}-E}{k_{b} T}\right)} .
\end{aligned}
$$

The conductivity under a steady state uniform generation rate is

$$
\sigma=q\left(\mu_{n} n+\mu_{p} p\right)
$$

where $n$ and $p$ are the total concentrations of electrons and holes in extended states, respectively. The photoconductivity is defined as 


$$
\sigma_{p h}=q\left[\mu_{n}\left(n-n_{e}\right)+\mu_{p}\left(p-p_{e}\right)\right]
$$

where $n_{e}$ and $p_{e}$ are the electron and hole concentrations in thermal equilibrium, respectively.

The ambipolar diffusion length is given by

$$
L_{a m b}=\sqrt{\frac{2 k_{b} T \mu_{n} \mu_{p}\left(n-n_{e}\right)\left(p-p_{e}\right)}{G\left[\mu_{n}\left(n-n_{e}\right)+\mu_{p}\left(p-p_{e}\right)\right]}} .
$$

The electronic occupation function of monovalent states under a steady state uniform generation rate is [39]

$$
f^{j}(E)=\frac{N^{j}}{N^{j}+P^{j}}, \quad j=V B T, C B T
$$

where $N^{j}$ y $P^{j}$ are

$$
\begin{array}{ll}
N^{j}=c_{n}^{j} n+c_{p}^{j} N_{V} e^{\frac{E_{V}-E}{k_{b} T}}, & j=V B T, C B T, \\
P^{j}=c_{p}^{j} p+c_{n}^{j} N_{C} e^{\frac{E-E_{C}}{k_{b} T}}, & j=V B T, C B T .
\end{array}
$$

The occupation functions of amphoteric states under a steady state uniform generation rate are [40]:

$$
\begin{aligned}
& f^{+}(E)=\frac{P^{0} P^{-}}{N^{+} N^{0}+N^{+} P^{-}+P^{0} P^{-}}, \\
& f^{-}(E)=\frac{N^{0} N^{+}}{P^{-} N^{+}+N^{0} N^{+}+P^{0} P^{-}}, \\
& f^{0}(E)=1-f^{-}-f^{+},
\end{aligned}
$$

where $N^{+}, N^{0}, P^{0}$ and $P^{-}$are defined by

$$
\begin{aligned}
& N^{+}=c_{n}^{+} n+2 c_{p}^{0} N_{V} \exp \left(\frac{E_{V}-E}{k_{b} T}\right), \\
& N^{0}=c_{n}^{0} n+\frac{c_{p}^{-}}{2} N_{V} \exp \left(\frac{E_{V}-E-U}{k_{b} T}\right), \\
& P^{0}=c_{p}^{0} p+\frac{c_{n}^{+}}{2} N_{C} \exp \left(\frac{E-E_{C}}{k_{b} T}\right), \\
& P^{-}=c_{p}^{-} p+2 c_{n}^{0} N_{C} \exp \left(\frac{E+U-E_{C}}{k_{b} T}\right) .
\end{aligned}
$$

When hopping is taken into account, the conductivity will be the sum of the conductivity via extended states and the conductivity by hopping. Equation (A5) will change to [19]

$$
\sigma=q\left(\mu_{n} n+\mu_{p} p\right)+\frac{q^{2}}{6 k_{b} T} \int_{E_{V}}^{E_{C}} N(E) f(E) \int_{E_{V}}^{E_{C}} \Gamma_{\mathrm{E}, \mathrm{E}^{\prime}} N\left(E^{\prime}\right)\left[1-f\left(E^{\prime}\right)\right] r_{E^{\prime}}^{2} d E^{\prime} d E,
$$


with

$$
\Gamma_{\mathrm{E}, \mathrm{E}^{\prime}}=\frac{v_{0}}{\int_{E_{V}}^{E} N\left(E^{\prime}\right) d E^{\prime}} \exp \left(-\frac{2 r_{E}}{R_{0}}\right)
$$

and

$$
\mathrm{r}_{\mathrm{E}}=\left[\int_{E_{V}}^{E} N\left(E^{\prime}\right) d E^{\prime}\right]^{-\frac{1}{3}}
$$

where $v_{0}$ is the attempt-to-hop frequency and $R_{0}$ the decay length of the wavefunction. From equation (A18) it can be observed that the contribution of hopping to the conductivity decreases as temperature increases, being negligible for temperatures higher than about $110 \mathrm{~K}$ according to different authors. $[18,19]$.

\section{Appendix B -Approximate analytical solutions}

The charge neutrality equation can be written as

$$
p+p^{\mathrm{VBT}}+p^{\mathrm{DB}}=n+n^{\mathrm{CBT}}+n^{\mathrm{DB}},
$$

while the continuity equation can be written as

$$
G-\left(n p-N_{C} N_{V} e^{\frac{E_{V}-E_{C}}{k_{b} T}}\right)\left(R^{C B T}+R^{V B T}+R^{D B}\right)=0
$$

We will first give approximate expressions for the terms involving monovalent states (VBT and CBT) and then for those involving amphoteric states (DB). The charges trapped in the conduction and valence band tails are

$$
\begin{aligned}
& n^{\mathrm{CBT}}=\int_{E_{V}}^{E_{C}} f^{C B T}(E) N_{C B T}(E) d E, \\
& p^{\mathrm{VBT}}=\int_{E_{V}}^{E_{C}}\left(1-f^{V B T}(E)\right) N_{V B T}(E) d E,
\end{aligned}
$$

where $f^{C B T}$ and $f^{V B T}$ represent the electronic occupation function of monovalent states, and are given by equation (A8). The occupation functions for the conduction and valence band tails can be well approximated by

$$
\begin{aligned}
& f^{C B T}(E) \cong \frac{n}{\left(n+p / r^{C B T}\right)\left(1+\exp \left(\frac{E-E_{t n}^{C B T}}{k_{b} T}\right)\right)}, \quad \text { for } E_{t p}^{C B T}<E<E_{C}, \\
& 1-f^{\mathrm{VBT}}(E) \cong \frac{p}{\left(r^{V B T} n+p\right)\left(1+\exp \left(\frac{E_{t p}^{V B T}-E}{k_{b} T}\right)\right)}, \quad \text { for } E_{V}<E<E_{t n}^{\mathrm{VBT}},
\end{aligned}
$$


where $E_{t n}^{j}$ and $E_{t p}^{j}$ are the quasi-Fermi levels for electrons and holes trapped in monovalent states, and $r^{j}=c_{n}^{j} / c_{p}^{j}, j=V B T$ or $C B T$. By definition, the quasi-Fermi levels for trapped charge are

$$
\begin{array}{ll}
E_{t n}^{j}=E_{C}+k_{b} T \ln \left(\frac{\mathrm{n}+\mathrm{p} / r^{j}}{N_{C}}\right), & j=V B T, C B T, \\
E_{t p}^{j}=E_{V}-k_{b} T \ln \left(\frac{r^{j} \mathrm{n}+\mathrm{p}}{N_{V}}\right), & j=V B T, C B T .
\end{array}
$$

Replacing equations (B5) and (B6) into equations (B3) and (B4), respectively, we get

$$
\begin{gathered}
n^{\mathrm{CBT}} \cong \frac{n I_{C B T} N_{C B T}\left(E_{t n}^{C B T}\right)}{n+p / r^{C B T}}, \\
p^{\mathrm{VBT}} \cong \frac{p I_{V B T} N_{V B T}\left(E_{t p}^{V B T}\right)}{r^{V B T} n+p},
\end{gathered}
$$

where $I_{C B T}$ and $I_{V B T}$ represent the following integrals:

$$
\begin{aligned}
& I_{C B T}=\int_{E_{t p}^{C B T}}^{E_{C}} \frac{e^{\frac{E-E_{t n}^{C B T}}{k_{b}{ }^{T} C}} d E}{1+e^{\frac{E-E_{t n}^{C B T}}{k_{b} T}}} \cong k_{b} T_{C}\left[e^{-0.2 \frac{T}{T_{C}}}+\frac{\left(\frac{N_{C}}{n+p / r^{C B T}}\right)^{\left(\frac{T}{T_{C}}-1\right)}-e^{0.8\left(\frac{T}{T_{C}}-1\right)}}{1-\frac{T_{C}}{T}}\right], \\
& I_{V B T}=\int_{E_{V}}^{E_{t n}^{C B T}} \frac{e^{\frac{E_{t p}^{V B T}-E}{k_{b} T_{V}} d E}}{1+e^{\frac{E_{t p}^{V B T}-E}{k_{b} T}}} \cong \int_{-\infty}^{+\infty} \frac{e^{\frac{E_{t p}^{V B T}-E}{k_{b} T V}} d E}{1+e^{\frac{E_{t p}^{V B T}-E}{k_{b} T}}}=\frac{\pi k_{b} T}{\sin \left(\frac{\pi T}{T_{V}}\right)}
\end{aligned}
$$

The analytical approximation (B12) is theoretically valid for $T<T_{V}$, but only results in a good approximation of the integral when the temperature is less than $\sim 75 \%$ of the characteristic temperature $T_{v}$. In a-Si:H $T_{V}$ is usually higher than $400 \mathrm{~K}$ [4], justifying the applicability of the approximation in the measured temperature range. $T_{C}$ is usually much smaller than $T_{V}$, consequently an approximation equivalent to equation (B12) would have a very narrow temperature validity. The deduction of the more complex analytical approximation (B11) is presented in appendix C, and we have numerically tested that its error is lower than $1 \%$ in the measured temperature range. A detailed deduction of equation (B12) can be found in Ref. [27].

The terms corresponding to the recombination in the conduction and valence band tails are

$$
\begin{aligned}
& R^{C B T}=\int_{E_{V}}^{E_{C}} \frac{c_{n}^{C B T} c_{p}^{C B T} N_{C B T}(E)}{N^{C B T}+P^{C B T}} d E, \\
& R^{V B T}=\int_{E_{V}}^{E_{C}} \frac{c_{n}^{V B T} c_{p}^{V B T} N_{V B T}(E)}{N^{V B T}+P^{V B T}} d E,
\end{aligned}
$$

where $N^{C B T}, N^{V B T}, P^{C B T}, P^{V B T}$ are defined in equations (A9) and (A10) [39]. The factors that multiply the DOS in the integrands of equations (B13) and (B14) can be approximated by 


$$
\begin{array}{ll}
\frac{c_{n}^{C B T} c_{p}^{C B T}}{N^{C B T}+P^{C B T}} \cong \frac{c_{p}^{C B T}}{\left(n+p / r^{C B T}\right)\left(1+\exp \left(\frac{E-E_{t n}^{C B T}}{k_{b} T}\right)\right)}, \quad \text { for } E_{t p}^{C B T}<E<E_{C}, \\
\frac{c_{n}^{V B T} c_{p}^{V B T}}{N^{V B T}+P^{V B T}} \cong \frac{c_{p}^{V B T}}{\left(n+p / r^{V B T}\right)\left(1+\exp \left(\frac{E_{t p}^{V B T}-E}{k_{b} T}\right)\right)}, \quad \text { for } E_{V}<E<E_{t n}^{\mathrm{VBT}} .
\end{array}
$$

Replacing equations (B15) and (B16) into equations (B13) and (B14), respectively, gives

$$
\begin{aligned}
& R^{C B T}=\int_{E_{V}}^{E_{C}} \frac{c_{n}^{C B T} c_{p}^{C B T} N_{C B T}(E)}{N^{C B T}+P^{C B T}} d E \cong \frac{c_{n}^{C B T} I_{C B T} N_{C B T}\left(E_{t n}^{C B T}\right)}{r^{C B T} n+p}, \\
& R^{V B T}=\int_{E_{V}}^{E_{C}} \frac{c_{n}^{V B T} c_{p}^{V B T} N_{V B T}(E)}{N^{V B T}+P^{V B T}} d E \cong \frac{c_{n}^{V B T} I_{V B T} N_{V B T}\left(E_{t p}^{V B T}\right)}{r^{V B T} n+p} .
\end{aligned}
$$

The negative and positive charges trapped in the deep states are given by

$$
\begin{aligned}
& n^{D B}=\int_{E_{V}}^{E_{C}} f^{-}(E) N_{D B}(E) d E, \\
& p^{D B}=\int_{E_{V}}^{E_{C}} f^{+}(E) N_{D B}(E) d E,
\end{aligned}
$$

where $f^{-}(E)$ and $f^{+}(E)$ give the probabilities of a DB center at energy $E$ to be negatively charged and positively charged, respectively. Their definitions are presented in equations (A12) and (A11). As a result of the slower variation with energy of the deep states concentrations, stronger approximations can be made on their occupation functions. Following the analysis made in Ref. [22], we have

$$
\begin{gathered}
f^{-}(E) \cong\left\{\begin{array}{cl}
1, & \text { for } E_{V}<E<E_{t p}^{0} \\
\frac{n}{n+p / r^{-}}, & \text {for } E_{t p}^{0}<E<E_{t p}^{+} \\
\frac{r^{+} r^{-} n^{2}}{r^{+} r^{-} n^{2}+r^{+} n p+p^{2}}, & \text { for } E_{t p}^{+}<E<E_{t n}^{-} \\
0, & \text { for } E_{t n}^{-}<E<E_{C}
\end{array}\right. \\
f^{+}(E) \cong\left\{\begin{array}{cl}
0, & \text { for } E_{V}<E<E_{t p}^{+} \\
\frac{p^{2}}{r^{+} r^{-} n^{2}+r^{+} n p+p^{2}}, & \text { for } E_{t p}^{+}<E<E_{t n}^{-} \\
\frac{p}{r^{+} n+p}, & \text { for } E_{t n}^{-}<E<E_{t n}^{0} \\
1, & \text { for } E_{t n}^{0}<E<E_{C}
\end{array}\right.
\end{gathered}
$$

where $r^{-}=c_{n}^{0} / c_{p}^{-}$and $r^{+}=c_{n}^{+} / c_{p}^{0}$. The quasi-Fermi levels for trapped-charge corresponding to amphoteric states are

$$
\begin{aligned}
& E_{t p}^{0}=E_{V}-U-k_{b} T \ln \left(2 \frac{r^{-} n+p}{N_{V}}\right), \\
& E_{t n}^{-}=E_{C}-U+k_{b} T \ln \left(\frac{n+p / r^{-}}{2 N_{C}}\right),
\end{aligned}
$$




$$
\begin{aligned}
& E_{t p}^{+}=E_{V}-k_{b} T \ln \left(\frac{r^{+} n+p}{2 N_{V}}\right) \\
& E_{t n}^{0}=E_{C}+k_{b} T \ln \left(2 \frac{n+p / r^{+}}{N_{C}}\right) .
\end{aligned}
$$

The approximations given by equations (B21) and (B22) are good while the temperature is not too high, and are exact at $0 \mathrm{~K} . E_{t p}^{0}$ could be smaller than $E_{V}$, falling outside the energy domain. In this case, the first line of equation (B21) should be neglected and we should make $E_{t p}^{0}=E_{V}$ in the second line to stay in the domain. See Ref. [22] for a complete discussion of DB quasi-Fermi levels. Replacing the approximations (B21) and (B22) into equations (B19) and (B20), respectively, we obtain

$$
\begin{gathered}
n^{D B} \cong \sum_{i} \frac{N_{D B}^{i}}{2}\left[\frac{p}{r^{-} n+p} \operatorname{erf}\left(\frac{E_{t p}^{0}-E_{D B}^{i}}{\omega_{i} \sqrt{2}}\right)-\operatorname{erf}\left(\frac{E_{V}-E_{D B}^{i}}{\omega_{i} \sqrt{2}}\right)+\frac{r^{-} n}{r^{+} r^{-} n^{2}+r^{+} n p+p^{2}}\left(\begin{array}{c}
r^{+} n \operatorname{erf}\left(\frac{E_{t n}^{-}-E_{D B}^{i}}{\omega_{i} \sqrt{2}}\right) \\
+\frac{p^{2}}{r^{-} n+p} \operatorname{erf}\left(\frac{E_{t p}^{+}-E_{D B}^{i}}{\omega_{i} \sqrt{2}}\right)
\end{array}\right)\right], \\
p^{D B} \cong \sum_{i} \frac{N_{D B}^{i}}{2}\left[\operatorname{erf}\left(\frac{E_{C}-E_{D B}^{i}}{\omega_{i} \sqrt{2}}\right)-\frac{n}{n+p / r^{+}} \operatorname{erf}\left(\frac{E_{t n}^{0}-E_{D B}^{i}}{\omega_{i} \sqrt{2}}\right)-\frac{p}{r^{+} r^{-} n^{2}+r^{+} n p+p^{2}}\left(\begin{array}{c}
p \operatorname{erf}\left(\frac{E_{t p}^{+}-E_{D B}^{i}}{\omega_{i} \sqrt{2}}\right) \\
+\frac{r^{-} n^{2}}{n+p / r^{+}} \operatorname{erf}\left(\frac{E_{t n}^{-}-E_{D B}^{i}}{\omega_{i} \sqrt{2}}\right)
\end{array}\right)\right],
\end{gathered}
$$

where we have used the identity for the definite integral of a normalized Gaussian function

$$
\int_{E_{1}}^{E_{2}} \frac{1}{\sqrt{2 \pi} \omega} \exp \left(\frac{-\left(E-E_{0}\right)^{2}}{2 \omega^{2}}\right) d E=\frac{1}{2}\left[\operatorname{erf}\left(\frac{E_{2}-E_{0}}{\omega \sqrt{2}}\right)-\operatorname{erf}\left(\frac{E_{1}-E_{0}}{\omega \sqrt{2}}\right)\right]
$$

The dangling bond term of equation (B2) is

$$
R^{D B}=\int_{E_{V}}^{E_{C}} \frac{\left(c_{n}^{+} c_{p}^{0} P^{-}+c_{n}^{0} c_{p}^{-} N^{+}\right) N_{D B}(E)}{N^{+} N^{0}+N^{+} P^{-}+P^{0} P^{-}} d E
$$

where $N^{+}, N^{0}, P^{0}$ y $P^{-}$are Okamoto's parameters [40], defined by equations (A14), (A15), (A16) and (A17). The factor multiplying the DOS inside the integrand of equation (B30) can be well approximated by the following formula, as soon as the temperature is not too high:

$$
\frac{\left(c_{n}^{+} c_{p}^{0} P^{-}+c_{n}^{0} c_{p}^{-} N^{+}\right)}{N^{+} N^{0}+N^{+} P^{-}+P^{0} P^{-}} \cong\left\{\begin{array}{cl}
0, & \text { for } E_{V}<E<E_{t p}^{+} \\
\frac{c_{n}^{+} p+c_{n}^{0} r^{+} n}{r^{+} r^{-} n^{2}+r^{+} n p+p^{2}}, & \text { for } E_{t p}^{+}<E<E_{t n}^{-} \\
\frac{c_{n}^{+}}{r^{+} n+p}, & \text { for } E_{t n}^{-}<E<E_{t n}^{0} \\
0, & \text { for } E_{t n}^{0}<E<E_{C}
\end{array}\right.
$$

Replacing equation (B31) into equation (B30), and using the property (B29), we finally obtain 


$$
R^{D B} \cong \sum_{i} \frac{N_{D B}^{i}}{2}\left[\begin{array}{c}
\frac{c_{n}^{+}}{r^{+} n+p} \operatorname{erf}\left(\frac{E_{t n}^{0}-E_{D B}^{i}}{\omega_{i} \sqrt{2}}\right)-\frac{c_{n}^{+} p+c_{n}^{0} r^{+} n}{r^{+} n\left(r^{-} n+p\right)+p^{2}} \operatorname{erf}\left(\frac{E_{t p}^{+}-E_{D B}^{i}}{\omega_{i} \sqrt{2}}\right) \\
+\frac{n}{n+p / r^{+}}\left(\frac{c_{n}^{0}\left(r^{+} n+p\right)-c_{n}^{+} r^{-} n}{r^{+} n\left(r^{-} n+p\right)+p^{2}}\right) \operatorname{erf}\left(\frac{E_{t n}^{-}-E_{D B}^{i}}{\omega_{i} \sqrt{2}}\right)
\end{array}\right] .
$$

\section{Appendix C - Approximation of integral $I_{C B T}$.}

$$
\begin{gathered}
\text { Defining } x=\frac{E-E_{t n}^{C B T}}{k_{b} T} \text { and } a=\frac{T}{T_{C}} \text {, equation (B11) can be rewritten as } \\
\qquad I_{C B T}=k_{b} T \frac{\int_{E_{t p}^{C B T}-E_{t n}^{C B T}}^{\frac{E_{C}-E_{t n}^{C B T}}{k_{b} T}}}{k_{b} T} f(x) e^{a x} d x,
\end{gathered}
$$

where $f(x)$ is:

$$
f(x)=\frac{1}{1+e^{x}}
$$

For $x$ large enough (see figure 7), the one in the denominator of equation (C2) can be discarded,

$$
f(x) \cong e^{-x},
$$

while for $x$ small enough (see below), a Maclaurin series could be used to approximate $f(x)$ :

$$
f(x) \cong 1-e^{x}
$$

The factor multiplying $f(x)$ inside the integrand is a fast growing $x$ function, consequently the largest $x$ values have the strongest contribution to the integral. Therefore, it is not necessary to have a very precise approximation of $f(x)$ for small $x$ values, and is enough to use just the first term of equation (C4).

According to the previous analysis, we can divide the integral (C1) in three regions:

$$
I_{C B T} \cong k_{b} T\left[\int_{\frac{E_{t p}^{C B T}-E_{t n}^{C B T}}{k_{b} T}}^{-b} e^{a x} d x+\int_{-b}^{c} f(x) e^{a x} d x+\int_{C}^{\frac{E_{C}-E_{t n}^{C B T}}{k_{b} T}} e^{(a-1) x} d x\right]
$$

where the first and third integrals can be solved analytically. The number $-b$ correspond to the $x$ value under which $f(x)=1$ results a good approximation. Similarly, the number $c$ corresponds to the $x$ value after which the approximation (C3) is a good one. From figure 7, where we plot $f(x)$ with the two mentioned approximations, we could choose $b=5$ and $c=3$. However, the problem is still unsolved because we are not able to evaluate the second integral of equation (C5). Its integration region $[-b, c]$ is much smaller than those corresponding to the other two integrals, consequently its contribution to the integral is minor. If we discard this term, the integral (C5) would be slightly underestimated. On the other hand, we can see in figure 7 that both approximations tend to overestimate $f(x)$ outside its validity range, i.e., the smaller we take $b$ and $c$, the higher is the overestimation of the integral. Consequently, 
discarding the second integral of equation (C5) could be compensated by extending the limits of the first and third integrals.

Discarding the middle integral and solving the other two, we have

$$
I_{C B T} \cong k_{b} T_{C}\left[e^{\left(\frac{-b T}{T_{C}}\right)}-e^{\left(E_{t p}^{C B T}-E_{t n}^{C B T}\right) / k_{b} T_{C}}+\frac{e^{\left(\frac{1}{k_{b} T_{C}}-\frac{1}{k_{b} T}\right) \cdot\left(E_{C}-E_{t n}^{C B T}\right)}-e^{c\left(\frac{T}{T_{C}}-1\right)}}{1-\frac{T_{C}}{T}}\right] .
$$

It have been found numerically that with $b=0.2$ and $c=0.8$ we obtain a very good estimation of the integral. The second term inside the brackets can be discarded, while the third term can be rewritten using equation (B7), finally giving

$$
I_{C B T} \cong k_{b} T_{C}\left[e^{-0.2 \frac{T}{T_{C}}}+\frac{\left(\frac{N_{C}}{n+p / r C B T}\right)^{\left(\frac{T}{T_{C}}-1\right)}-e^{0.8\left(\frac{T}{T_{C}}-1\right)}}{1-\frac{T_{C}}{T}}\right] .
$$

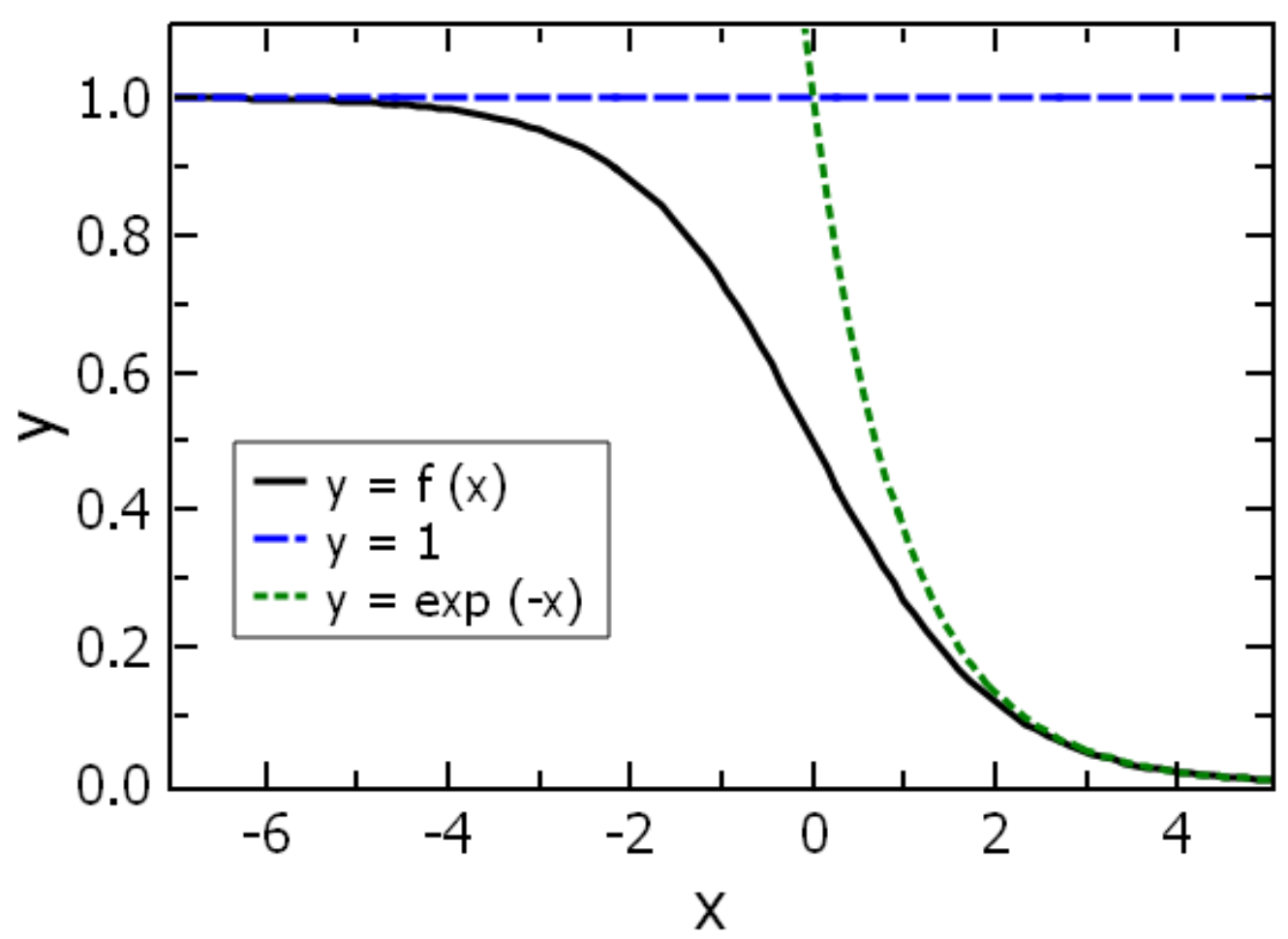

Figure 7. Function $(\mathrm{C} 2)$ is plotted in black, together with the asymptotic approximations valid for small $x$ values (in blue) and for large $x$ values (in green). 


\section{References}

[1] Ovshinsky S R and Madan A 1978 Nature 276482.

[2] Street R A 2000 Technology and Applications of Amorphous Silicon (Springer-Verlag, Berlin Heidelberg).

[3] Fang J, Yan B, Li T, Wei C, Huang Q, Chen X, Wang G, Hou G, Zhao Y, Zhang X 2018 Sol. Energy Mater. Sol. Cells 176167.

[4] Street R A 1991 Hydrogenated Amorphous Silicon (Cambridge University Press, Cambridge).

[5] Cohen M H, Fritzsche H and Ovshinsky S R 1969 Phys. Rev. Lett. 22, 1065.

[6] Balberg I, Dover Y, Naides R, Conde J P and Chu V 2004 Phys. Rev. B 69035203.

[7] Powell M J and Deane S C 1993 Phys. Rev. B 4810815.

[8] Schumm G 1994 Phys. Rev. B 492427.

[9] Fantoni A, Viera M and Martins R 2002 Sol. Energy Mater. Sol. Cells 73151.

[10] Vicente A T, Wojcik P J, Mendes M J, Águas H, Fortunato E and Martins R 2017 Solar Energy 144 232.

[11] Lang D V, Cohen J D and Harbison J P 1982 Phys. Rev. B 25, 5285.

[12] A recent review can be found in: Reynolds S, Brinza M, Benkhedir M L and Adriaenssens G J 2017 Photoconductivity in Materials Research, in Springer Handbook of Electronic and Photonic Materials, edited by S. Kasap and P. Capper, (Springer International Publishing) Chapter 7, pp. 151174.

[13] Main C, Berkin J and Merazga A 1991 Photoconductivity in amorphous semiconductors Experiment and computer modeling, in Proceedings of the Sixth International School of Condensed Matter Physics, Varna, edited by M. Borissov, N. Kirov, J.M. Marshall, and A. Vavrék (World Scientific, Singapore) p. 55.

[14] Patel S J, Panchal A K and Kheraj V 2014 Applied Energy 119384.

[15] Rao R V, Savsani V J and Vakharia D P 2011 Computer-Aided Design 43303.

[16] Shklovskii B I, Levin E I, Fritzsche H and Baranovskii S D 1990 Advances in Disordered Semiconductors Vol. 3, edited by H Fritzsche (Singapore: World Scientific) p. 161.

[17] Bodurtha K and Kakalios J 2015 J. Appl. Phys. 118, 215103.

[18] Merazga A, Tobbeche S, Main C, Al-Shahrani A and Reynolds S 2006 J. Phys.: Condens. Matter 18 3721.

[19] Longeaud C and Tobbeche S 2009 J. Phys.: Condens. Matter 21045508. 
[20] Ritter D, Zeldov E and Weiser K 1986 Appl. Phys. Lett. 49791.

[21] Vanecek M and Poruba A 2002 Applied Physics Letters 80719.

[22] Longeaud C. and Kleider J P 1993 Phys. Rev. B 488715.

[23] Stutzmann M and Jackson W B 1987 Solid State Commun. 62153.

[24] Willemen J A 1998 Modelling of Amorphous Silicon Single- and Multi-Junction Solar Cells (Phd. thesis Delft University of Technology).

[25] O'Leary S K, Johnson S R and Lim P K 1997 Journal of Applied Physics 823334.

[26] Furlan J, Smole F and Popovic P 1993 Mat. Res. Soc. Symp. Proc. 297 363-368.

[27] Kopprio L, Longeaud C and Schmidt J A 2017 Journal of Applied Physics 122085702.

[28] Fritzsche H 2001 Annual Review of Material Research 3147.

[29] Fath Allah A, Ventosinos F and Longeaud C 2014 J. Phys.: Conf. Series 558012011.

[30] Schmidt J A and Longeaud C 2005 Phys. Rev. B 71125208.

[31] Cody G D, Tiedje T, Abeles B, Brooks B and Goldstein Y 1981 Phys. Rev. Lett. 471480.

[32] Weiser G and Mell H 1989 Journal of Non-Crystalline Solids 114298.

[33] Longeaud C and Kleider J P 1992 Phys. Rev. B 4511672.

[34] Longeaud C and Tobbeche S 2009 J. Phys.: Condens. Matter 21045508.

[35] Street R A, Zesch J and Thompson M J 1983 Appl. Phys. Lett. 43672.

[36] Street R A 1984 Philos. Mag. B 49 L15.

[37] Stutzmann M 1992 Philos. Mag. Lett. 66147.

[38] Longeaud C and Schmidt J A 2012 J. Non-Cryst. Solids 3582052.

[39] Halpern V 1986 Philos. Mag. B 54473.

[40] Okamoto H, Kida H and Hamakawa Y 1984 Philos. Mag. B 49231. 